

\title{
Association Of Vitamin D Deficiency With Risk Factors In Postmenopausal Women
}

\author{
N.M. Inoyatova \\ Associate Professor, PhD, Center For The Development Of Professional Skills Of Medical \\ Staff» Of The Ministry Of Health Of The Republic Of Uzbekistan, Department Of Obstetrics, \\ Gynecology And Perinatal Medicine, Uzbekistan
}

Copyright: Original

content from this work may be used under the terms of the creative commons attributes 4.o licence.

\section{ABSTRACT}

In conditions of age-related decrease in sex hormones and a number of pathological conditions and diseases in postmenopausal women, there is a deficiency of D hormone. In our country, the geographic location of which is below northern latitude and sufficient ultraviolet radiation, an insufficient content of vitamin $D$ is seen in postmenopausal women. There are a lot of risk factors leading to vitamin $D$ deficiency - the presence of smog and dust in cities, insufficient consumption of vitamin-fortified foods, the presence of problems with the gastrointestinal tract and excretory system and a number of others. One of the important factors that reduce vitamin storage is overweight and obesity, especially in combination with old age, when all absorption processes are reduced. The aim of our research was to study risk factors in women with vitamin D deficiency with subsequent correction of the deficiency state. We examined the level of total $25(\mathrm{OH}) \mathrm{D}$ in the blood serum in 46 postmenopausal women, and identified risk factors. Vitamin D deficiency was detected in $86.96 \%$ of women, and its deficiency was registered in $10.87 \%$. At the same time, a pronounced vitamin deficiency was not registered in any patient. Overweight was registered in $32.6 \%$, obesity of varying degrees in 26.1\%. Given the indicators, recommendations were given for correcting vitamin D deficiency. All postmenopausal women, especially those with risk factors for deficiency, are recommended to determine the basic level of vitamin $\mathrm{D}$. In case of deficiency, drug correction is recommended to reduce the risk of cardiovascular and oncological diseases.

\section{KEYWORDS}

Vitamin D, postmenopausal women, risk factors, obesity, hypovitaminosis, correction 


\section{INTRODUCTION}

Vitamin D was recently known as a regulator of calcium-phosphorus metabolism. Recently, however, a lot of information has appeared on the effect of vitamin $D$ on other physiological parameters of the body, both women and men $[9,14]$. Vitamin $D$ is chemically similar to progesterone. Today it is considered a steroid hormone with progesterone-like activity and its effect on the body is great [3]. There is enough information in the literature on the effect on reproductive function - maintaining the luteal phase, ensuring endometrial receptivity, anti-inflammatory effect, as well as the effect on pregnancy - reducing the frequency of premature birth and late spontaneous miscarriages, low birth weight $[1,2,4,12]$. Given the association of vitamin $D$ deficiency with a decrease in fertility, as well as with a worsening of maternal and perinatal pregnancy outcomes, it is recommended to normalize its indicators in pregravid preparation $[4,10]$. There is more evidence that vitamin $D$ hypovitaminosis leads to an increase in the incidence of cancer and autoimmune diseases, diabetes mellitus, there is a connection with obesity, the development of these complications is most typical for older age. In recent years, it is increasingly recognized that vitamin $D$ has a significant effect on the functioning of the organs of the cardiovascular system. Epidemiological researches have shown that there is an inverse correlation between vitamin levels and diseases such as hypertension, atherosclerosis, coronary heart disease, myocardial infarction, heart failure, stroke and hyperlipidemia $[6,11]$. Prevention of the above complications reduces the incidence of postmenopausal problems. Vitamin $D$ deficiency is indeed a global problem.

\section{THE MAIN RESULTS AND FINDINGS}

Vitamin D in 80-90\% enters the body through the skin, a small part of it comes with food. There are varieties of vitamin D - D3 (cholecalciferol) and D2 (ergocalciferol). D3 (cholecalciferol) is called "solar" because it is formed in the skin through exposure to ultraviolet light. To do this, children and adults should be in the sun for about 15-30 minutes several times a week. However, the intense production of vitamin $D$ occurs during unhealthy hours - from 11:00 to 14:00, when the skin needs protection. Ultraviolet radiation from the sun is composed of UVA and UVB rays. Vitamin $D$ synthesis is more actively triggered in the presence of UVB radiation (although it is considered more aggressive, does not allow glass to pass through). D2 (ergocalciferol) enters the body along with the usual food; unfortunately, there are not many animal foods enriched with vitamin D [13].

Despite the fact that the geographical location of Uzbekistan is at 41 latitude, it is below 35 parallel north latitude; in a zone of moderate insolation, a sunny country, where there is plenty of ultraviolet radiation, but vitamin $\mathrm{D}$ deficiency is common. What is the reason for this? There are many reasons for the deficit in Uzbekistan. First of all, residents of large cities in the atmosphere have a large layer of smog and dust ("dust cushion") - this prevents UV rays from breaking through. Secondly, insufficient consumption of foods with polyunsaturated fatty acids (fatty fish). Thirdly, insufficient sun exposure, dark skin color, closed clothing are also risk factors for vitamin $D$ deficiency. Also, impaired absorption of vitamin $\mathrm{D}$ due to gastrointestinal diseases 
(malabsorption syndrome, Crohn's disease, pancreatitis, cholestasis, etc.), long-term use glucocorticoids, liver and kidney disease, when the processes of vitamin $D$ hydroxylation are disrupted $[7,8]$. Obesity is also a factor in which vitamin $D$ is deposited in adipose tissue. These risk factors are most often found in the older age group, respectively, the risk group is women over the age of 50 . Old age is also a risk factor for hypovitaminosis, this fact is associated with a decrease in the production of vitamin $D$ in the skin, absorption of vitamin $D$ in the intestine, the number of VDR receptors in muscle tissue and functional activity of the kidneys, which in turn aggravates the deficiency state.

Menopause is characterized by ovarian depletion, a decrease in the production of steroid hormones. About 1/3 of a woman's life falls on the period of menopause, on average it is at least 15-20 years. In turn, the menopausal age is characterized by certain problems: from early psychovegetative disorders, urogenital problems and changes in the skin to late cardiovascular disorders, osteoporosis and Alzheimer's disease [11]. For this age, the most common cause of death is coronary heart disease. Thus, the combination of the problems of menopausal age and vitamin D deficiency dictates the need to search for risk factors and timely correction in this age group.

Considering the above, we examined 46 postmenopausal women. The aim of our study was to study risk factors in women with vitamin $D$ deficiency with subsequent adequate correction of hypovitaminosis. The examination of women was carried out in the polyclinic of the City Perinatal Center.

\section{RESEARCH METHODS}

Assessment of the level of total $25(\mathrm{OH}) \mathrm{D}$ in blood serum. Determination of the quantitative $25-\mathrm{OH}$ vitamin $\mathrm{D}$ in blood serum was carried out by chemiluminescence immunoassay in vitro using an automatic chemiluminescence immunological analyzer of the MAGLUMI series. To interpret the results, we used the most frequently recommended standards for the concentration of $25(\mathrm{OH})$ vitamin D: an indicator up to $10 \mathrm{ng} / \mathrm{ml}(25 \mathrm{nmol}$ / I) is interpreted as a pronounced deficiency, $11-20 \mathrm{ng} / \mathrm{ml}$ (50 nmol / I) indicates a deficiency, 21-30 ng / ml (51-75 nmol / I) - insufficiency, with values over $30 \mathrm{ng} / \mathrm{ml}(75 \mathrm{nmol} / \mathrm{l})$, the concentration of vitamin $\mathrm{D}$ has an adequate level. For prevention and treatment, it is recommended to adhere to the target level in the range of 30-60 $\mathrm{ng} / \mathrm{ml}(75-150 \mathrm{nmol} / \mathrm{L})$.

\section{RESULTS OF THE RESEARCH}

The age of the examined women ranged from 49 to 70 years. The median age was 56.1 years. There were 8 elderly women (over 60 years old) in the study group (17.4\%), they are a risk group for severe vitamin $D$ deficiency. The menopause age ranged from 1 to 20 years. From the group of women examined, early menopause (40-45 years old) was diagnosed in 9 (19.6\%) women, in 1 (2.2\%) women at 56 years old, in the remaining 36 (78.3) women - timely. 
Table 1. Vitamin D-status of the studied women.

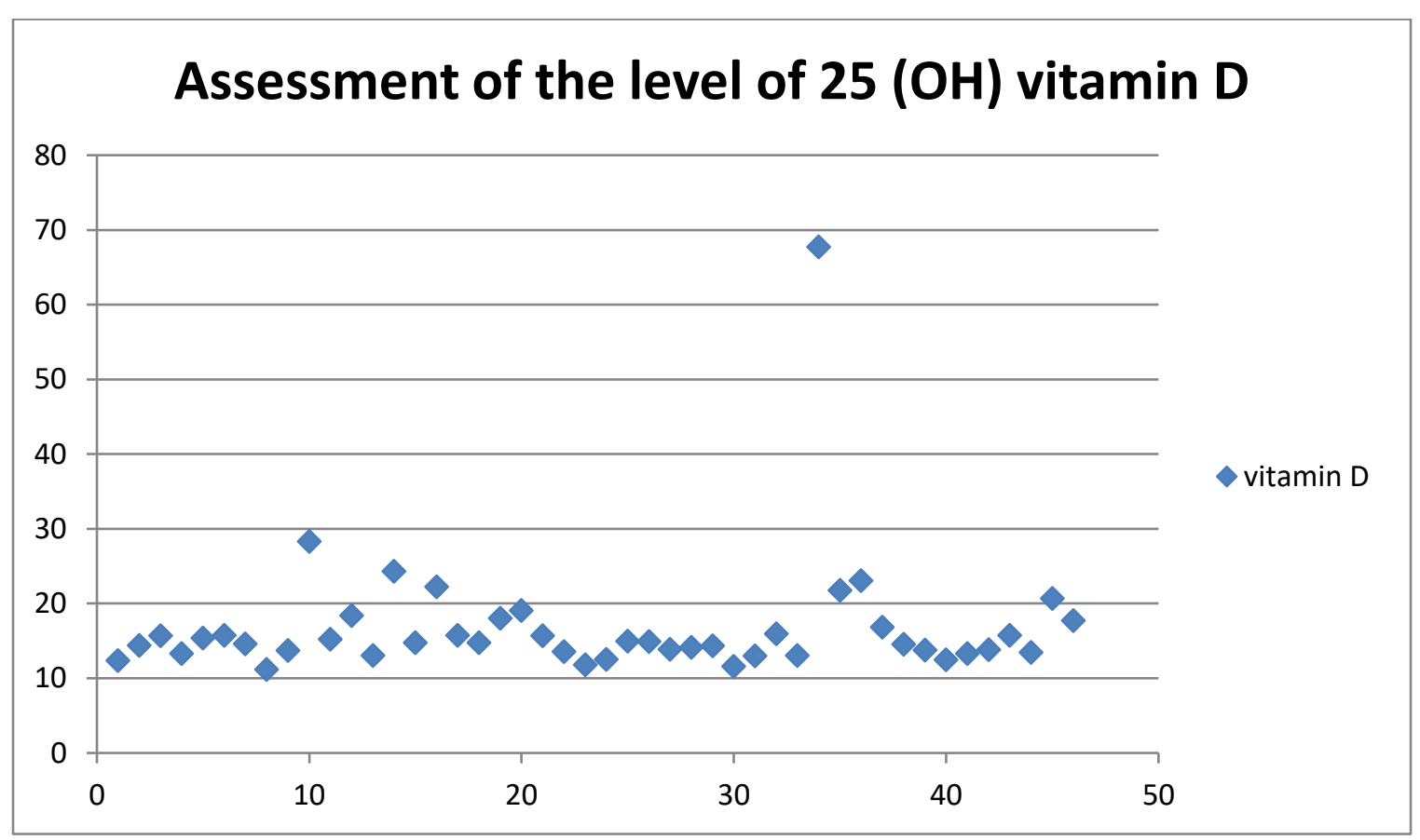

Determination of the level of total $25(\mathrm{OH}) \mathrm{D}$ in blood serum in 46 postmenopausal women revealed the following values (Table 1). An adequate level of vitamin $D$ of $67.77 \mathrm{ng} / \mathrm{ml}$ was registered only in a 53-year-old patient who had been taking drugs in a prophylactic dosage for the last 4 months. The remaining 45 women were diagnosed with decreased vitamin $D$ levels. The mean serum $25(\mathrm{OH})$ D levels in the deficient group were $16.39 \pm 3.87$, and in the deficient group - $23.96 \pm 2.4$.
We found that $97.8 \%$ of the women undergoing examination had reduced levels of vitamin $D$ in the blood serum (Table 2). Of these, vitamin D deficiency was registered in $10.87 \%$ of women in the surveyed group. At the same time, a pronounced vitamin deficiency was not registered in any patient from the group, which indicates the intake of vitamin into the body. In $86.96 \%$ of women, a deficiency of vitamin D in the blood serum was found, which corresponds to the idea of the frequent occurrence of a reduced vitamin D-status.

Table 2. Vitamin D-status by identified groups

\begin{tabular}{|c|c|c|}
\hline Serum 25 $(\mathrm{OH})$ D level & Number of people & $\%$ of all investigated \\
\hline Severe vitamin D deficiency & 0 & $0 \%$ \\
\hline Vitamin D deficiency & 40 & $86,96 \%$ \\
\hline Insufficiency of vitamin D & 5 & $10,87 \%$ \\
\hline Adequate vitamin D levels & 1 & $2,17 \%$ \\
\hline
\end{tabular}


The next stage of the examination was to identify risk factors that lead to vitamin $D$ deficiency. Given possible adjustments of risk factors, recommendations were given for correcting hypovitaminosis in order to further reduce complications in this category of women.

The risk group for vitamin $\mathrm{D}$ deficiency includes older age; people with a dark skin tone; other factors in which insufficient exposure to ultraviolet rays occurs. That is, these are factors in which, even with adequate availability or intake of "solar" vitamin, vitamin deficiency develops. It was said above that in the surveyed group, $17.4 \%$ of the deficiency state was associated with one of the factors, such as old age. 11 (23.9\%) women from the study group had a dark skin tone. The main source of vitamin $D$ is its formation under the influence of UV rays, which is aggravated by insufficient exposure to them associated with closed clothing. This fact was revealed in every second patient, in 24 (52.2\%) postmenopausal women.

Overweight and obesity also lead to an increased prevalence of vitamin D deficiency. Menopausal age is characterized by an increase in body weight due to reduced metabolism and insufficient physical activity. Since the termination of ovarian function in women, the number of women with metabolic syndrome has increased three times more. Obesity in menopause is accompanied by a decrease in fat-free mass, including muscle mass. Vitamin D deficiency is observed due to deposition in the fat mass, thus a vicious circle develops, which can only be broken with adequate correction of vitamin $D$ deficiency and weight loss. In the group of surveyed women, body mass index was normal (BMI 19.8-24.9) in 19 women (41.3\%), excess body weight (25-29.9) was found in 15 women (32.6\%). In 12 (26.1\%) women, obesity (30 or more) of varying degrees was registered. Moreover, class 1 obesity (BMI 30-34.9) was diagnosed in 8 (17.4\%) women, class 2 obesity (BMI 35-39.9) - in 3 (6.5\%) women, class 3 obesity (BMI 40-44.9) - in 1 (2.2\%) woman. Noteworthy is the fact that in the group of obese women, vitamin $\mathrm{D}$ values ranged from 11.8 to $14.36 \mathrm{ng} / \mathrm{ml}$, that is, all patients had vitamin $D$ deficiency. It is well known that obesity leads to an increased risk of metabolic and cardiovascular diseases, cancer, and this leads to an increased risk of mortality in this group. Researches show that weight loss is problematic when vitamin $D$ is deficient. According to the WHO, vitamin D deficiency increases the risk of cancers such as cancer of the endometrium, breast, ovaries, esophagus, stomach, liver, gallbladder, kidney, colon, Hodgkin's and non-Hodgkin's lymphomas $[4,14]$. In the surveyed group, 2 women were registered with breast cancer, 1 woman with non-Hodgik's lymphoma, which amounted to 6.5\%. The vitamin $\mathrm{D}$ concentration in these patients showed a deficiency of 14.36 to 17.76 $\mathrm{ng} / \mathrm{ml}$.

By the time of examination, in the study group of women, the consequences of both climacteric age and hypovitaminosis $D$ were found: arterial hypertension - 14 (in 30.4\% of cases), stroke and heart attack - 1 case (respectively, $2.2 \%$ in each case), diabetes mellitus - 2 (4.3\%). Diseases of the thyroid gland (hypothyroidism, nodular goiter) and the respiratory system have also been reported. The clinical parameters of hypovitaminosis were assessed. Clinical symptoms of vitamin D deficiency have no obvious manifestations and 
are scarce. Most often, women are concerned about isolated or generalized pain, discomfort in bones and muscles. Vitamin D deficiency leads to myopathy, which can manifest itself as muscle weakness, especially in the proximal muscle groups, diffuse muscle pain, and difficulty walking. In the group of surveyed women, the above complaints of varying severity from mild to severe were observed in 37 patients, which amounted to $80.4 \%$.
When studying the anamnesis in this category of women, it shows a number of problems in both obstetric-gynecological and somatic status (Table 3). Problems in reproductive health were observed in $65.2 \%$ of women (uterine myoma, fibroadenoma, fibrocystic breast disease, secondary infertility). A burdened obstetric history was observed in $54.3 \%$ of women, in the form of hypertensive conditions, postpartum hemorrhage, placental abruption, antenatal fetal death.

Table 3. Assessment of patient anamnestic data

\begin{tabular}{|c|c|c|c|}
\hline $\begin{array}{c}\text { Burdened gynecological } \\
\text { anamnesis }\end{array}$ & \% of women & $\begin{array}{c}\text { Complicated obstetric } \\
\text { anamnesis }\end{array}$ & \% of women \\
\hline Uterine fibroid & $45,6 \%(21)$ & Non-developing pregnancy & $15,2 \%(7)$ \\
\hline Pelvic inflammatory diseases & $13 \%(6)$ & Premature birth & $17,4 \%(8)$ \\
\hline Sterility & $10,9 \%(5)$ & Intrauterine fetal death & $4,3 \%(2)$ \\
\hline Tumor diseases of the ovaries & $10,8 \%(5)$ & Preeclampsia & $47,8 \%(22)$ \\
\hline Cervical disease & $6,5 \%(3)$ & Cervical insufficiency & $10,8 \%(5)$ \\
\hline Fibrocystic mastopathy & $58,7 \%(27)$ & Placental abruption & $19,6 \%(9)$ \\
\hline Fibroadenoma (operated on) & $4,3 \%(2)$ & Postpartum hemorrhage & $23,9 \%(11)$ \\
\hline
\end{tabular}

All patients with vitamin $\mathrm{D}$ deficiency were offered drug correction, the recommended drug was cholecalciferol (vitamin $D_{3}$ ). The proposed schemes for correcting vitamin $D$ deficiency were selected individually, depending on the initial level and the achievement of target values. The recommended serum $25(\mathrm{OH}) \mathrm{D}$ level is $40-60$ $\mathrm{ng} / \mathrm{ml}$ (100-150 $\mathrm{nmol} / \mathrm{L})$. Table 3 presents an algorithm that can be used to select the optimal daily dose of vitamin $D$ preparations depending on its initial serum level and the desired one $[8,10]$. Prescribing the drugs was taking into account the contraindications to taking vitamin D preparation. The patient with normal vitamin levels was advised to continue prophylactic doses of the vitamin. 
Table 3. Average change in blood concentration based on consumption per day (IU / day).

\begin{tabular}{|c|c|c|c|c|c|c|}
\hline \multicolumn{2}{|c|}{ Expected level, $\mathrm{ng} / \mathrm{ml}$} & 20 & 30 & 40 & 50 & 60 \\
\hline & & \multicolumn{5}{|c|}{ The recommended daily amount of vitamin $\mathrm{D}_{3}$, IU } \\
\hline \multirow{7}{*}{  } & 10 & 2000 & 4000 & 6000 & 10000 & 10000 \\
\hline & 15 & 1000 & 3000 & 6000 & 9000 & 10000 \\
\hline & 20 & & 2000 & 5000 & 8000 & 10000 \\
\hline & 25 & & 1000 & 4000 & 7000 & 10000 \\
\hline & 30 & & & 3000 & 6000 & 10000 \\
\hline & 35 & & & 1000 & 5000 & 9000 \\
\hline & 40 & & & & 5000 & 9000 \\
\hline
\end{tabular}

\section{CONCLUSION}

1. All postmenopausal women, especially those with risk factors for vitamin D deficiency, are recommended to determine total $25(\mathrm{OH}) \mathrm{D}$ in serum.

2. All postmenopausal women with overweight and obesity are recommended to correct hypovitaminosis and weight loss.

3. All postmenopausal women with insufficient vitamin $D$ content are recommended drug correction to reduce the risk of cardiovascular and oncological diseases.

4. The role of vitamin $D$ in the prevention of complications in postmenopausal age requires further study on the basis of more clinical material.

\section{REFERENCES}

1. Bakleicheva M.O., Kovaleva I.V., Bespalova O.N., Kogan I.Yu. The effect of vitamin $D$ on a woman's reproductive health // Journal of Obstetrics and
Women's Diseases. - 2018. - T. 67. - No. 3. P. 4-19.

2. Gromova OA, Torshin I.Yu., Dzhijikhia LK, Gogoleva I.V. The role of vitamin D in the prevention and treatment of female infertility. Gynecology. 2016; 18 (3): 3439.

3. Dobrokhotova Yu.E., Borovkova E.I., Zalesskaya S.A. et al. Vitamin D3 and women's health. Gynecology. 2019; 21 (1): 44-51.

4. Clinical guidelines. Vitamin D deficiency in adults: diagnosis, treatment and prevention. Moscow 2016 page 39

5. Kozak K.V. The role of vitamin D deficiency in the development of pathology in perimenopausal women HEALTH OF WOMAN. 2018.5 (131): 76-79;

6. Morozova Yu.E., Tarasova M.A. Physiological role of vitamin $\mathrm{D}$ and the importance of its deficiency in the pathogenesis of climacteric syndrome. 2018. - T. 67. - No. 3. - P. 74-82.

7. Nurlyagayanov R.Z., Sirtlanova E.R. The prevalence of vitamin $D$ deficiency in people over 50 years old, permanently 
residing in the Republic of Bashkortostan, during the period of minimal insolation. Osteoporosis and Osteopathy. 2012; 15 (3): 7-9.

8. Ozolinya L.A., Savchenko T.N., Safonina M.S., Overko A.V. The importance of vitamin $D$ for the health of perimenopausal women. Medical advice. 2020; (13): 84-90.

9. Pigarova E.A., Petrushkina A.A. Nonclassical effects of vitamin D. Osteoporosis and osteopathy. 2017; 20 (3): 90-101

10. Shikh E.V., Tikhomirov S.V., Zaitseva T.A., Segedina E.M., Treyvish L.S. Analysis of the effectiveness of the use of various dosage regimens and forms of cholecalciferol in patients with reproductive dysfunction // Doktor.Ru. 2019. No. 4 (159). S. 54-58.

11. Yachinskaya T.V. Menopausal hormone therapy and vitamin $D$ in postmenopausal women: quality of life today and tomorrow. S. 28-31. https: //cyberleninka.ru/

12. Al-Jaroudi D, Al-Banyan N, Aljohani NJ, Kaddour O. Vitamin D deficiency among subfertile women: a case-control study. Gynecol Endocrinol. 2016; 32 (4): 272-275

13. Grass roots Health Moving Research into Practice [Electronic resource]. - Access mode: http://www.grassrootshealth.net/

14. Michael F. Holick, Neil C. Binkley, Heike A. Bischoff-Ferrari, Catherine M. Gordon, David A. Hanley, Robert P. Heaney, M. Hassan Murad, Connie M. Weaver Evaluation, treatment, and prevention of vitamin $D$ deficiency: an Endocrine Society clinical practice guideline. J Clin Endocrinol Metab. 2011 Jul; 96 (7): 19111930, doi: 10.1210 / jc.2011-0385 